\title{
Zero dimensional exciton-polaritons
}

\author{
A. Baas ${ }^{*}{ }^{1}$, O. El Daïf ${ }^{1}$, M. Richard ${ }^{1}$, J.-P. Brantut ${ }^{1}$, G. Nardin $^{1}$, R. Idrissi Kaitouni ${ }^{1}$, \\ T. Guillet ${ }^{2}$, V. Savona ${ }^{3}$, J. L. Staehli ${ }^{1}$, F. Morier-Genoud ${ }^{1}$, and B. Deveaud ${ }^{1}$ \\ 'Institut de Photonique et d'Électronique Quantiques (IPEQ) École Polytechnique Fédérale de Lausanne \\ (EPFL), 1015 Lausanne, Switzerland \\ 2 Groupe d'étude des Semiconducteurs (GES), Université de Montpellier II, Place Eugène Bataillon, \\ 34095 Montpellier cedex 5, France \\ ${ }^{3}$ Institut de théorie des phénomènes physiques (ITP) École polytechnique fédérale de Lausanne (EPFL), \\ 1015 Lausanne, Switzerland
}

Received 17 March 2006, revised 24 April 2006, accepted 24 April 2006

Published online 17 July 2006

PACS 42.65.-k, 71.35.Lk, 71.36.+c

We present a novel semiconductor structure in which $0 \mathrm{D}$ polaritons coexist with $2 \mathrm{D}$ microcavity polaritons. Spatial trapping of the 2D microcavity polaritons results from the confinement of their photonic part in a potential well, consisting of an adjustable thickness variation of the spacer layer. This original technique allows to create polaritonic boxes of any size and shape. Strong coupling regime is evidenced by the typical energy level anticrossing, in real space and in momentum space, and supported by a theoretical model.

Semiconductor heterostructures allow the analysis of light-matter interaction in nanoscopic or mesoscopic systems, in which properties of both uncoupled light and matter oscillators can be strongly engineered. Beyond the motivation of the realization of optoelectronic devices, monitoring of light by matter and vice versa has already led to a wide range of new fascinating physics. Of particular interest is the strong coupling regime, where the dressed states basis becomes the relevant one to describe and understand the coupled system. Historic experiments of quantum electrodynamics have been realized using a few atoms in the strong coupling regime with a few cavity photons [1,2]. In strong analogy, three groups have recently reported the successful achievement of strong coupling regime for one single Quantum Dot (QD) with a high-quality factor (Q) cavity mode [3-5]. In these systems the small number of electronic excitations opens the way to the realization of solid state devices for quantum information operation.

Research on solid state systems has also been motivated, during the last 50 years, by the possibility to achieve Bose-Einstein condensation (BEC) at high temperature. This effect involves a huge number of particles, that massively accumulate into a single quantum state below a critical temperature, thus displaying macroscopical quantum properties. To reach this goal, the most promising candidates are microcavity polaritons, eigenstates of semiconductor microcavities in strong coupling regime [6]. These quasiparticles have the great advantage over excitons to exhibit a very light effective mass. Their bosonic behavior at low density has been demonstrated already by final state stimulation in parametric scattering process [7], and by direct evidence of quantum degeneracy [8]. Rather high temperatures have been reached for such effects [9]. However, polariton BEC has not been demonstrated yet. A more favorable situation for BEC should be obtained by confining the polaritons within a small volume, as suggested by

* Corresponding author: e-mail: augustin.baas@epfl.ch 
the recent observation of localized polaritons under non resonant pumping [10] and several theoretical works (see in particular [11]).

In this paper we demonstrate the successful confinement of polaritons along the three directions of space, through the confinement of the photonic component of the 2D microcavity polaritons. Photon confinement is obtained through local variations of the thickness of the microcavity spacer layer. Thanks to the light effective mass of polaritons, a lateral confinement on the micron scale is sufficient to achieve quantization of the polariton states, with a step height of the order of several nanometers. In contrast with the technique based on the etching of the whole upper Bragg mirror and spacer [12,13], the 2D cavity is preserved, thus allowing the $2 \mathrm{D}$ polaritons surrounding the mesa to interact with the $0 \mathrm{D}$ ones. Moreover the lateral losses due to this confinement are intrinsically negligible with this technique, which is remaining an important limitation to strong coupling in the precedent cases [14]. It also allows to control at will the number of discrete confined states and their energy spacing, by tuning the height of the trap and its lateral size. Finally, the absence of constraints for the geometry and size of the mesas opens the way to experiments involving polaritons of any shape and dimensionality.

We chose to manufacture $6 \mathrm{~nm}$ high circular mesas with diameters ranging from $3 \mu \mathrm{m}$ to $19 \mu \mathrm{m}$. The height has to be chosen once for the whole sample, as it is determined by the sample processing. The final structure, including circular mesas, is schematically represented on Fig. 1(a). It is fabricated by Molecular Beam Epitaxy (MBE), with an intermediate photolithography step in order to etch the mesas on the top surface of the cavity spacer layer. Let us note that the quantum well exciton mode is not affected at all by the etching process, whatever the pattern of the mesa (For more details see [15]).

In order to check the energy depth of our mesas, we performed reflectivity measurements on large square mesas of $300 \mu \mathrm{m}$ before growing the upper Bragg mirror. The energy difference between the cavity modes with and without a mesa was found to be $9 \mathrm{meV}$. AFM measurements were then performed on the final structure, and surprisingly the very small thickness variation of the mesa, etched on the top of the spacer layer, is conserved despite the growth of the 2.5 micrometer thick upper Bragg mirror. The regrowth resulted in the structure represented on the scheme of the sample on Fig. 1(a). However, as shown by Fig. 1(d) the edge steepness is much more pronounced along the crystalline axis [011] than $\left[\begin{array}{ll}0 & 1\end{array}\right]$. This asymmetry is also visible on the small circular mesas shown in Fig. 1(b) and (c) even though the lateral sizes match the expected value.

This asymmetrical shape is due to the anisotropic mobility of the atoms on the surface during the growth. So it slowly builds-up during the growth process. The interface sharpness of the steps slowly smoothen along the growth, the extension being maximum on the last pairs of the top mirror. As the cavity mode field extends only over the first pairs of the mirror (closest to the spacer layer [16]), the optical properties of the mesas will be weakly affected by this effect. On the other hand another source of shape asymmetry is the photolithography mask, where the patterned mesas, instead of being perfect circles, are slightly elliptical. This asymmetry does affect the optical properties of the mesa: it is responsible for a degeneracy lifting in the mesa emission spectrum. Similar observations have recently been re-
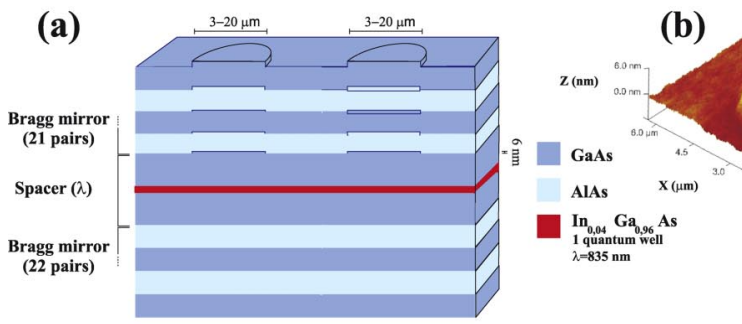

(c)

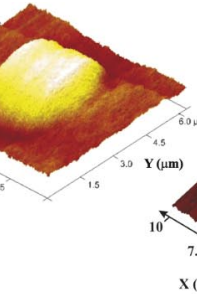

(d)

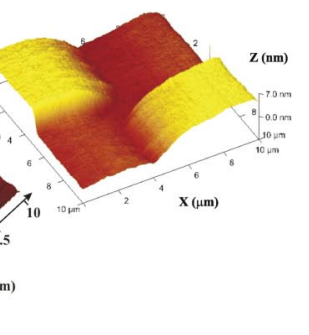

Fig. 1 (online colour at: www.pss-b.com) (a) Scheme of the sample. Only the first layers of the Bragg mirrors are represented. (b-d) AFM image on the surface on the top Bragg mirror. (b) AFM image of a $3 \mu \mathrm{m}$-diameter quasicircular mesa. (c) AFM image of a $9 \mu \mathrm{m}$-diameter quasi-circular mesa. (d) AFM image of a junction between two $300 \mu \mathrm{m}$-side square mesas. 
ported for QDs embedded in similar mesas by Ref. [17], although the growth induced asymmetry seems to be much more pronounced, probably due to different growth conditions.

A clear evidence of the strong coupling regime can only be given by the observation of an anticrossing behavior between the system dressed states energies. In QDs systems, the exciton energy is tuned across the cavity mode by acting on the sample's temperature. The thickness variation along the radius of our sample allows us to tune the cavity length by choosing a specific position along this wedge. The exciton energy of the quantum well is almost not affected by this thickness variation and remains stable across the whole sample.

The sample is cooled to $10 \mathrm{~K}$, and non-resonantly excited by an Argon laser focused on a $30 \mu \mathrm{m}$ or $2 \mu \mathrm{m}$ diameter spot. The luminescence is injected into a $25 \mu \mathrm{eV}$ resolution spectrometer. The setup can be easily exploited for real-space or momentum $(\boldsymbol{k}$-)space spectrally resolved imaging of the luminescence. To do so, the desired image (real plane or Fourier plane) is formed on the entrance slit of the monochromator. The entrance slit selects a narrow stripe in the image which is then dispersed along the horizontal axis by the monochromator to obtain the spectral information for each point of the stripe. Of particular interest is the spectrally resolved momentum space imaging which provides directly the luminescence in the dispersion plane $(E,|\boldsymbol{k}|)$ (see Figs. 2(c), 3 and 4).

In the investigated sample the 2D microcavity exhibits a $3.5 \mathrm{meV}$ Rabi splitting, to be compared to the $220 \mu \mathrm{eV}$ Full Width at Half Maximum (FWHM) of the bare 2D cavity mode and the $500 \mu \mathrm{eV}$ of the quantum well exciton. On Fig. 2(a), we plot the luminescence energy of each discrete state of the $3 \mu \mathrm{m}$ diameter mesas versus their position along the sample's wedge. The strong coupling regime in the mesas is unambiguously demonstrated by this measurement: a clear energy anticrossing is displayed by the three couples of confined upper and lower polariton states. They exhibit $a \approx 3.35 \mathrm{meV}$ Rabi splitting, slightly smaller than in the $2 \mathrm{D}$ cavity. The linewidth of the confined ground cavity mode reaches $70 \mu \mathrm{eV}$, yielding a $Q$-factor as high as 21000 [18]. As discussed previously, a degeneracy lifting due to the elliptic shape of the mesas is visible for the first and second excited lower polariton states at negative detunings $\left(\delta=E_{\text {cav }}-E_{\text {exciton }}\right)$. However, this splitting is hardly resolved for the upper polariton states, their linewidth being much larger because of the coupling to $2 \mathrm{D}$ continuum and to $0 \mathrm{D}$ lower energy states.
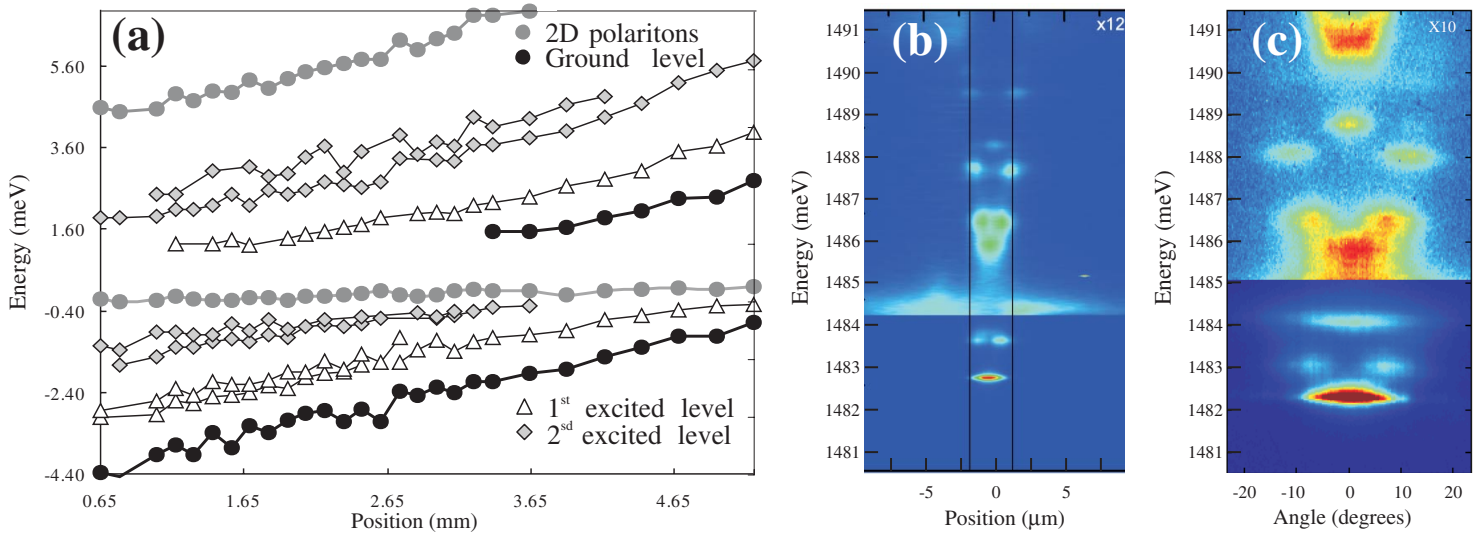

Fig. 2 (online colour at: www.pss-b.com) (a) Energy of the photoluminescence lines as a function of the position along the sample's wedge for a $3 \mu \mathrm{m}$-diameter mesa (the zero of the energy scale corresponds to the exciton energy). Rabi splitting of $3.5 \mathrm{meV}(3.35 \mathrm{meV})$ for the $2 \mathrm{D}$ polaritons (0D polaritons). Zero detuning for the ground level around $4.6 \mathrm{~mm}$., for the first excited level around $3.0 \mathrm{~mm}$ and the for second excited level around $1.9 \mathrm{~mm}$. (b) Photoluminescence intensity as a function of energy and position for a $3 \mu \mathrm{m}$-diameter quasi-circular mesa. For clarity, the intensity above $1484.2 \mathrm{meV}$ has been multiplied by a constant factor, as indicated. (c) Photoluminescence intensity as a function of energy and emission angle for a $3 \mu \mathrm{m}$-diameter quasi-circular mesa. For clarity, the intensity above $1485 \mathrm{meV}$ has been multiplied by a constant factor, as indicated. 

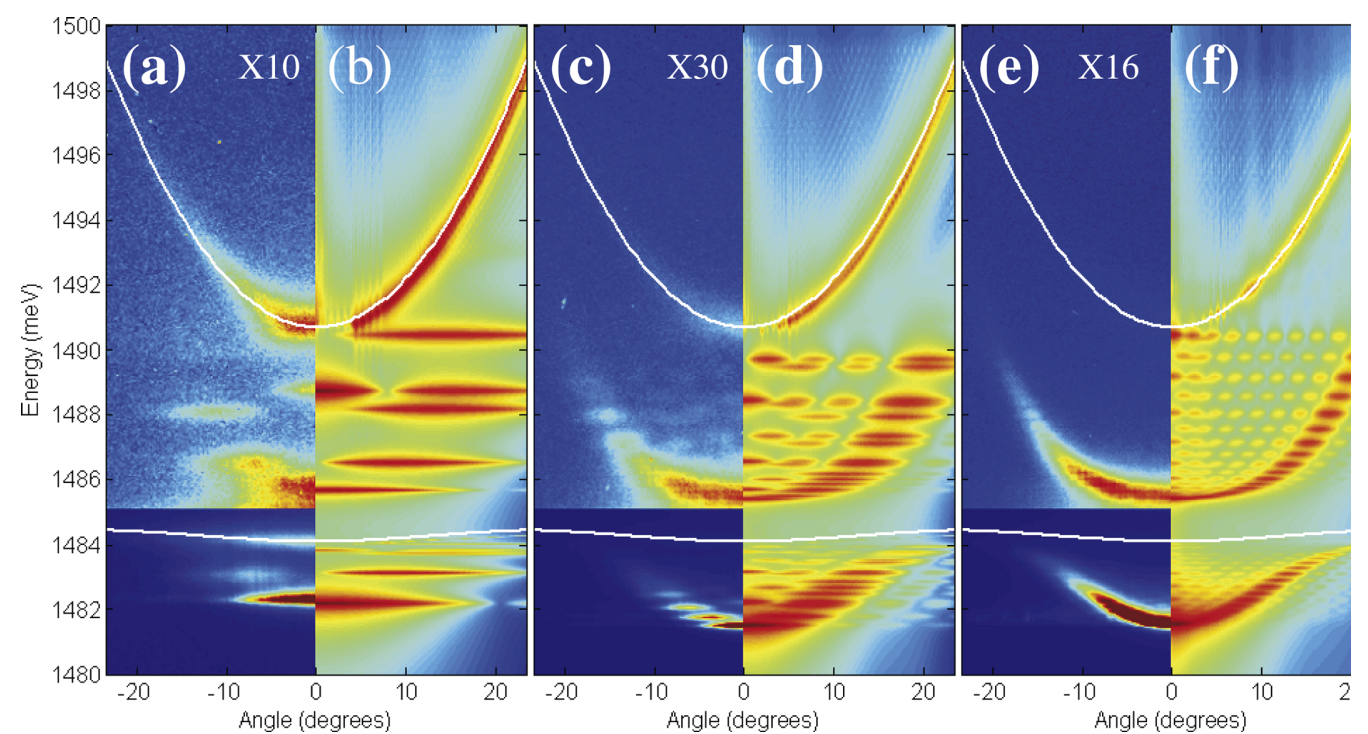

Fig. 3 (online colour at: www.pss-b.com) Measured photoluminescence intensity as a function of energy and emission angle for a $3 \mu \mathrm{m}$-diameter (a), $9 \mu \mathrm{m}$-diameter (c) and $19 \mu \mathrm{m}$-diameter (e) quasi-circular mesa. White lines: energy dispersions of 2D polaritons modes. For clarity, intensities above $1485 \mathrm{meV}$ have been multiplied by a constant factor, as indicated. Intensity plot of the simulated polariton spectral density for a $3 \mu \mathrm{m}$ (b), $9 \mu \mathrm{m}$ (d), $19 \mu \mathrm{m}$ (f) cylindrical mesa.

The strong coupling being clearly demonstrated, we got interested into characterizing the $0 \mathrm{D}$ polaritons in real and reciprocal space. Figure 2(b) is a spatially resolved spectrum [19] of a $15 \mu \mathrm{m}$ stripe of the sample surface (horizontal axis) containing a $3 \mu \mathrm{m}$ diameter mesa, non-resonantly excited by a $2 \mu \mathrm{m}$ diameter laser spot. The stripe axis was chosen perpendicular to the wedge in order to prevent non-intrinsic

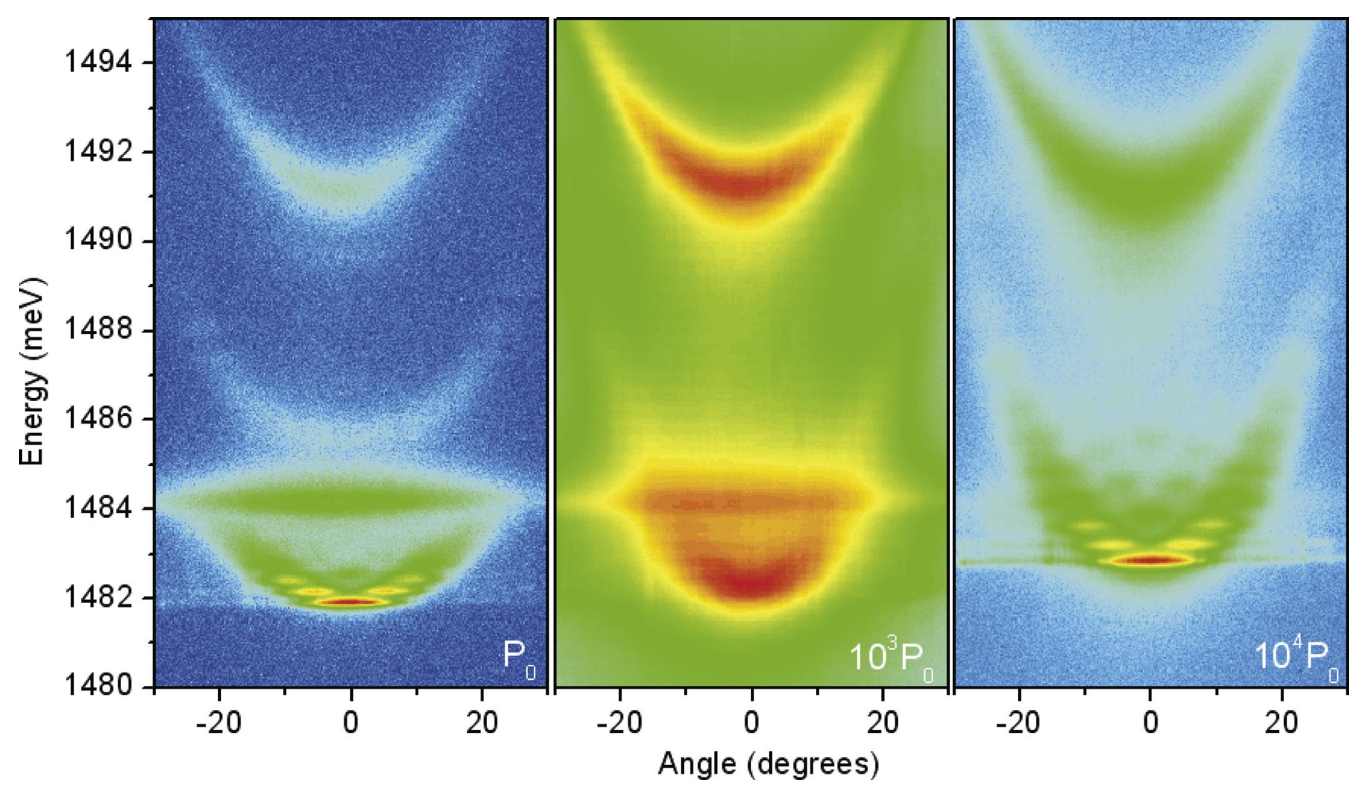

Fig. 4 (online colour at: www.pss-b.com) Photoluminescence intensity as a function of energy and emission angle for a $9 \mu \mathrm{m}$-diameter quasi-circular mesa for three different pump densities: $P_{0}=5 \mu \mathrm{W} \mathrm{cm}{ }^{-2}$ (a), $10^{3} P_{0}$ (b) and $10^{4} P_{0}$ (c). For clarity, the contrast has been adjusted for each pump density. 
sources of asymmetry. The luminescence emitted around the mesa (delimited by the black vertical lines) at $1483.8 \mathrm{meV}$ and $1490.5 \mathrm{meV}$ originates from the $2 \mathrm{D}$ upper and lower polaritons at a positive detuning of $\delta=E_{\text {cav }}-E_{\text {exc }} \approx 2 \Omega_{\mathrm{R}}$. Note that the luminescence part of these modes situated inside the mesa exhibits much weaker intensity than outside. These intensity "holes" can most probably be attributed to the efficient coupling between the 2D and 0D polariton states coexisting inside the mesa. The states localized within the mesa can clearly be attributed to the lower and the upper 0D polaritons states. As expected from a basic calculation of the extension of a wave function confined in a potential well, the ground level exhibits one centered lobe, whereas the first excited states present two symmetrical lobes, both visible on the corresponding lower and upper polariton. In addition to the lift of degeneracy, note that the asymmetrical shape of the mesa also results in a dependance of the luminescence on the in-plane direction. This is the reason why the luminescence intensity is not always symmetric with respect to the mesa axis. The acquisition of spectrally resolved images with two real space dimensions are under progress, in order to give a 2D mapping of each $0 \mathrm{D}$ polariton state wavefunction, as already realized for pure photonic modes in [18].

The observation of the luminescence in the (energy, angle) plane provides many interesting information as well. Such a measurement performed on a $3 \mu \mathrm{m}$ mesa is shown on Fig. 2(c). In the dispersion plane, for a usual 2D microcavity, a peculiarly shaped dispersion curve is caused by the anticrossing behaviour between the pure exciton and the pure cavity modes. This dispersion shape attests that the cavity is in strong coupling regime. However, in the case of $0 \mathrm{D}$ polaritons in small mesas, as expected, the polariton dispersion is flat and quantized down to few pairs of discrete upper and lower polaritons states. So the energy anticrossing is hard to identify, it is much easier for larger mesas, as seen on Fig. 3(c) and (e).

In the $3 \mu \mathrm{m}$ mesa of Fig. 2(c), the quantization of the dispersion produces discrete spectrally narrow lines, extended over 20 degrees along the momentum axis. The ground and first excited lower polariton states appear below the exciton resonance, while four states are visible for the upper polariton. The wide angular extension of the emission is related consistently to the $3 \mu \mathrm{m}$ confinement diameter. Note that if one does not take into account the effect of the small asymmetry of the mesas, the spectrally resolved luminescence intensities in real and momentum space are directly the squared modulus of the wave function radial dependance, simply related one to the other by a Fourier transform. The extended 2D polariton modes are visible as well on these figures, and exhibit the expected continuous dispersion. To overcome their relatively weak intensity, the corresponding theoretical dispersion have been superimposed with white lines.

The identification of the different discrete levels of the confined polaritons is validated by the comparison with a semi-classical model exposed in the contribution of P. Lugan et al., in the present issue [20]. This calculation provides the energy and momentum optical density of the 0D polariton states of a circular mesa. As can be seen in Fig. 3 a very good quantitative agreement with the measurements is achieved, whatever the size of the mesa. The intensity in the theoretical plots (Fig. 3(b-e)) should not be compared with the experimental ones: the computed value being the optical density, it does not account for any relaxation process. The details of this calculation can be found in Ref. [20].

We so far mainly addressed the $3 \mu \mathrm{m}$ diameter mesas, but measurements were also performed for larger mesas, namely with diameters of 9 and $19 \mu \mathrm{m}$. The experimental dispersion curves are shown on Fig. 3(c) and (e). As expected the number of confined levels increases with the size of the mesa, and they get closer energetically. For the largest mesa a quasi-continuous dispersion can be seen. The asymmetry of the mesa probably explains the slight energy discrepancy between the simulation and the measurement in the smallest mesas.

As a definitive proof of the occurrence of the strong coupling regime in the mesa, we observe a drastic change of the emission spectrum when increasing the excitation intensity from the polaritonic linear regime to weak coupling regime. The experiment is realized on a cylindrical $9 \mu \mathrm{m}$ mesa and presented on Fig. 4 using the dispersion curve. Figure 4(a) was taken at the lowest excitation power $\left(5 \mu \mathrm{W} \mathrm{cm}{ }^{-2}\right)$, where the energy and linewidth of the polariton states are that of the linear regime. The excitation conditions are identical to that of Fig. 3(c). Increasing the excitation intensity by three orders of magnitudes, 
we obtain the strongly modified pattern represented on Fig. 4(b). The lower states luminescence is blue shifted by $0.4 \mathrm{meV}$ and strongly broadened, while their dispersion now crosses the excitonic level. The loss of the anticrossing in the dispersion plane attests for the loss of the strong coupling regime, which is caused by saturation of the exciton transition. Then the excitation power is increased by another order of magnitude (Fig. 4(c)). Only the photonic modes of the system are still visible. Therefore, although we did not observe yet collective effect of $0 \mathrm{D}$ polaritons, we are very confident that we will succeed by adding a few more quantum wells in the cavity in order to increase the oscillator strength.

In conclusion, we have unambiguously demonstrated that we have realized a new device of very high quality where polaritons are confined along the three directions of space. We have presented direct observation of the spatial trapping of 2D microcavity polaritons and discussed their peculiar properties. To go further, the complex dynamical properties of such structures needs to be understood by using time resolution and 2D real space imaging. Such type of devices opens the way to many possibilities, such as studies on interacting polaritons in coupled traps. It should also allow the achievement of polariton condensates within handmade traps of any shape and dimensionality.

Acknowledgements This work was supported by the Quantum Photonics NCCR and the Swiss National Science Foundation. We thank C. Ciuti, W. Langbein, A. Quattropani, M. Saba, and P. Schwendimann for fruitful discussions.

\section{References}

[1] M. Brune et al., Phys. Rev. Lett. 76, 1800 (1996).

[2] J. McKeever et al., Nature 425, 268 (2003).

[3] J. P. Reithmaier et al., Nature 432, 197 (2004).

[4] T. Yoshie et al., Nature 432, 200 (2004).

[5] E. Peter et al., Phys. Rev. Lett. 95, 067401 (2005).

[6] C. Weisbuch et al., Phys. Rev. Lett. 69, 3314 (1992).

[7] R. M. Stevenson et al., Phys. Rev. Lett. 85, 3680 (2000).

[8] A. Baas et al., Phys. Rev. Lett. 96, 176401 (2006).

[9] M. Saba et al., Nature 414, 731 (2001).

[10] M. Richard et al., Phys. Rev. B 72, 210310 (2005).

[11] D. Sarchi and V. Savona, see contribution on the present issue.

[12] G. Dasbach et al., Phys. Rev. B 64, 201309(R) (2001).

[13] G. Dasbach et al., Phys. Rev. B 65, 245316 (2002).

[14] M. Obert et al., Appl. Phys. Lett 84, 1435 (2004).

[15] O. El Daïf et al., Appl. Phys. Lett. 88, 061105 (2006).

[16] V. Savona et al., Phase Transit. 68, 169 (1999).

[17] D. Lu et al., Appl. Phys. Lett. 87, 163105 (2005).

[18] D. Sanvito et al., Appl. Phys. Lett. 86, 191109 (2005).

[19] H. Lohmeyer et al., Appl. Phys. Lett. 88, 051101 (2006).

[20] P. Lugan et al., see contribution on the present issue. 